Also the remarkable insensitivity of the results to major changes in nuclei and bonding must be accommodated by any overall theory of the phenomenon.

T. F. Hunter

M. C. R. Symons

Department of Chemistry,

University of Leicester.

Received February 13, 1967.

${ }^{1}$ Symons, M. C. R., J. Chem. Soc., 2276 (1965).

${ }^{2}$ Atkins, P. W., and Symons, M. C. R., The Structure of Inorganic Radicals, 3 (Elsevier, 1966).

${ }^{3}$ Gross, J. M., and Symons, M. C. R., J. Chem. Soc., A, 451 (1966).

\section{Method for estimating the Molecular Weight of Linear Polyethylene}

A METHOD for estimating the molecular weight of a long chain linear polyethylene filament is discussed. This method is based on the assumed chain configurations of the polymer before and after extrusion from a jet, and on the straightening of the chain of the filament in a load elongation experiment. This filament can be considered as partially oriented at extrusion (jet stretch). The degree of such orientation is largely related to the speed of extrusion and the speed of taking up the filament. A typical load-elongation curve of a high crystalline, linear polyethylene filament is shown in Fig. 1. "Necking" begins after a few per cent of elongation. The load is approximately a constant during necking. After the completion of necking the load increases with further extension until the filament breaks.

The high crystalline polymer is melted before extrusion and thus the chains are randomly coiled. Let $N$ be the average number of the $\mathrm{C}-\mathrm{C}$ bonds in the chain. Then the end to end distance of the randomly coiled chain, calculated from the principlo of random flight ${ }^{1}$, is $c(2 N)^{\frac{1}{2}}$ where $c$ is the $\mathrm{C}-\mathrm{C}$ bond length. After extrusion the polymer molecules recrystallize (assuming that the ratio of amorphous part to erystalline part is very small) and the segments of a chain are straightened in the crystalline regions. Let $n$ be the average number of the $\mathrm{C}-\mathrm{C}$ bonds in a straight segment. The average length of the straight segment is $n \cdot c \cdot \sin \theta$, where $2 \theta$ is the $\mathbf{C}-\mathrm{C}-\mathrm{C}$ angle $\left(2 \theta=112^{\circ}\right)$.

We first assume that each chain passes through a crystalline region without chain folding. The end to end

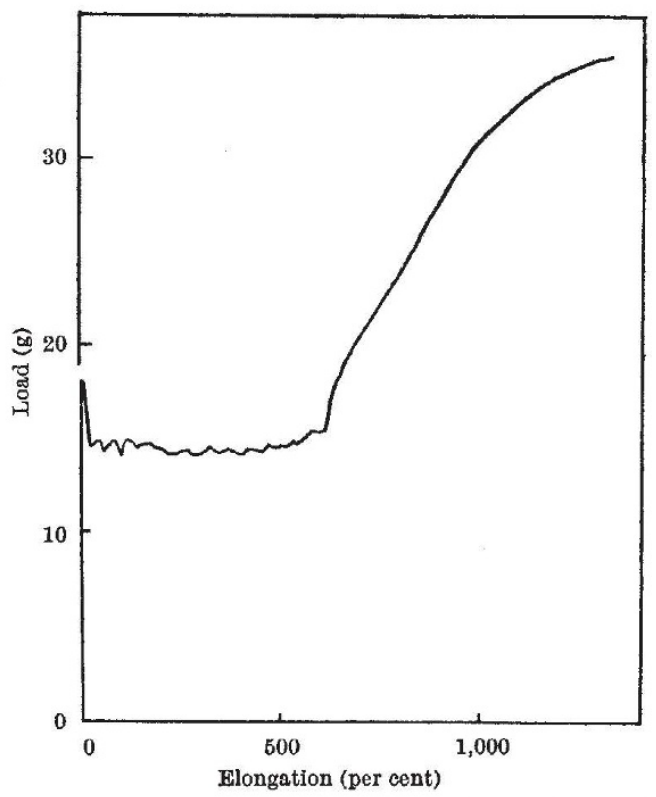

Fig. 1. Load-elongation curve of a linear polyethylene filament (linear density of the filament is $77 \mathrm{~g}$ per $9,000 \mathrm{~m}$ ). distance of a chain in the filament (assuming random orientation for the crystallites) is then given by

$$
n \cdot c \cdot \sin \theta \cdot(2 N / n)^{\frac{1}{2}}
$$

We may then define the per cent jet stretch $e_{1}$ as

$e_{j}=100\left[\frac{n c \cdot \sin \theta \cdot(2 N / n)^{\frac{1}{2}}}{c(2 N)^{\frac{1}{2}}}-1\right]=100\left[n^{\frac{1}{2}} \sin \theta-1\right]$

At the completion of necking in a load-elongation experiment, a chain which passes through a number of crystalline regions in the filament is approximately straightened without intermolecular sliding. Let $e_{n}$ be the per cent elongation at the completion of necking; we then have

$$
\begin{aligned}
& e_{n}=100\left[\frac{c N \sin \theta}{n c \cdot \sin \theta \cdot(2 N / n)^{\frac{1}{2}}}-1\right]= \\
& 100\left[\left(\frac{N}{2 n}\right)^{\frac{1}{2}}-1\right]
\end{aligned}
$$

Eliminating $n$ between equations (1) and (2) we obtain

$$
e_{j}+e_{n}\left(1+e_{j} / 100\right)=100\left[(N / 2)^{\frac{1}{2}} \sin \theta-1\right]
$$

Next we assume that the crystalline region is formed by chain folding. A single polymer chain in the filament can form a series of crystalline regions of random orientation. Let $L$ be the average number of times of ehain folding in a crystalline region. Assuming $L<n$ we then have

and

$$
\begin{aligned}
& e_{j} \simeq 100\left[\frac{n c \cdot \sin \theta \cdot(2 N / n L)^{\frac{1}{2}}}{c(2 N)^{\frac{1}{2}}}-1\right]= \\
& 100\left[(n / L)^{\frac{1}{2}} \sin \theta-1\right]
\end{aligned}
$$

$$
\begin{aligned}
& e_{n} \simeq 100\left[\frac{c N \sin \theta}{n c \cdot \sin \theta \cdot(2 N / n L)^{\frac{1}{2}}}-1\right]= \\
& 100\left[(N L / 2 n)^{\frac{1}{2}}-1\right]
\end{aligned}
$$

From equations (4) and (5), equation (3) is again obtained.

The equations can be used to calculate $N$ from estimated values of $e_{j}$ and $e_{n}$, or to calculate $e_{j}$ and $n$ (or the ratio $n / L)$ for a certain extrusion condition from known molecular weight of the polymer and estimated value of $e_{n}$. For the filament used to obtain Fig. 1, we estimate $e_{j} \simeq 300$ and $e_{n} \simeq 600$. From equation (3) we obtain $N \simeq 2,300$. The molecular weight of this sample is about 32,000 .

Part of the work was carried out at the Research Laboratory, American Viscose Corporation, Marcus Hook, Pennsylvania.

\section{Y. Liang}

Department of Physics,

San Fernando Valley State College,

Northridge, California.

Received January 30; revised February 14, 1967.

${ }^{1}$ Flory, P. J., Principles of Polymer Chemistry, 415 (Cornell University Press, Ithaca, 1953).

\section{BIOCHEMISTRY}

\section{Biochemical Evidence for a Distinct Type of Primary Gout}

In recent years several reports ${ }^{1-3}$ have called attention to a disorder occurring in the paediatric age group which is characterized by marked over-production of uric acid. The clinical features include haematuria and uric acid stone disease, and often, but not always, mental retardation, neurological signs, and self-mutilation. Intravenous injection of glycine ${ }^{14} \mathrm{C}$ into such patients results in a rapid incorporation ${ }^{1}$ of isotope into urinary urio acid, 